Int. J. Dev. Biol. 50: 309-314 (2006)

doi: $10.1387 /$ ijdb.052048ap

\title{
Spatial patterns formed by chemotactic bacteria Escherichia coli
}

\author{
ANDREY A. POLEZHAEV*,1 RUSLAN A. PASHKOV², ALEXEY I. LOBANOV² \\ and IGOR B. PETROV² \\ ${ }^{1}$ P.N. Lebedev Physical Institute, Moscow, Russia and ${ }^{2}$ Moscow Institute of Physics and Technology, Russia
}

\begin{abstract}
In certain experimental conditions, bacteria form complex spatial-temporal patterns. A striking example of such kind was reported by Budrene and Berg (1991), who observed a wide variety of different colony structures ranging from arrays of spots to radially oriented stripes or arrangements of more complex elongated spots, formed by Escherichia coli. We discuss the relevant mechanisms of intercellular regulation in bacterial colony which may cause pattern formation, and formulate the corresponding mathematical model. In numerical experiments a variety of patterns, observed in real systems, is reproduced. The dynamics of their formation is investigated.
\end{abstract}

KEY WORDS: bacterial colony, chemotaxis, spatial pattern, mathematical modeling

\section{Introduction}

There are numerous experimental observations of complex spatial-temporal patterns formed by bacterial colonies. One of the first evidence of this kind was reported by Houser (1885) more than a hundred years ago, who observed the formation of stable circular structures in Proteus colonies. In the present paper we discuss the mechanism of complex stationary pattern formation observed in Escherichia colicolonies under certain experimental conditions. Though we confine to this particular species, it seems obvious that similar generic mechanisms should manifest themselves in other morphogenetic events. The experiment, which we refer to, was performed by Budrene and Berg $(1991,1995)$, in which chemotactic strains of the bacterium $E$. coli were inoculated on semi-solid agar containing mixtures of amino acids or sugars. They found that cells could aggregate chemotactically, resulting in a wide variety of different colony structures ranging from arrays of spots to radially oriented stripes or arrangements of more complex elongated spots. Two of their figures are reproduced in Fig. 1. The spots or stripes are dense accumulations of cells visualized by scattered light. They arise sequentially in a wake of a spreading circular band, which first appeared at the edge of the inoculum and them migrated outwards at a constant speed. Every few hours, new sets of stripes or spots appeared in the wake.

These aggregates first appeared as faint inhomogeneities that rapidly intensified. They contained vigorously motile bacteria. Later on, the spots faded (scattered less light). At this stage all the cells in the aggregate were nonmotile. The patterns, generated by cells, are the result of their response to gradients of attractant which they excrete themselves. These patterns are far more elaborate than those observed when chemotactic strains grow on media containing nutrients that are attractants (Adler, 1966; Nossal, 1972; Wolfe and Berg, 1989; Agladse et al., 1993). They also differ from the well known traveling waves of aggregating cells of the slime mold Dictyostelium discoideum (Bonner, 1967) in that the structures formed by $E$. coli are temporary stable. However it is worth mentioning that this class of patterns is not limited to E. coli, similar structures were observed in Salmonella typhymurium (Woodward et al., 1995).

In many biological systems spatial pattern formation is often the result of the loss of stability of the initially uniform state. Mathematical models of such processes are usually the sets of partial differential equations of the reaction diffusion type (see, for example, Murray, 1989). Besides diffusion instability, an essential factor, which may promote pattern formation, is taxis, i.e. directed cell motion along the gradient of some chemical substance (chemotaxis) or mechanical stress gradient (haptotaxis). Examples of the corresponding models are the ones describing wound healing processes (Lauffenburger and Kennedy, 1983; Alt and Lauffenburger, 1987), aggregation of amoebae $D$. discoideum (Keller and Segel, 1970, 1971; Nanjundiah, 1973), models of patterns formed in the embryogenesis processes (Oster and Murray, 1989), models of pigmentation processes in alligators (Murray et al., 1990) and snakes (Murray and Myerscough, 1991) and others. 

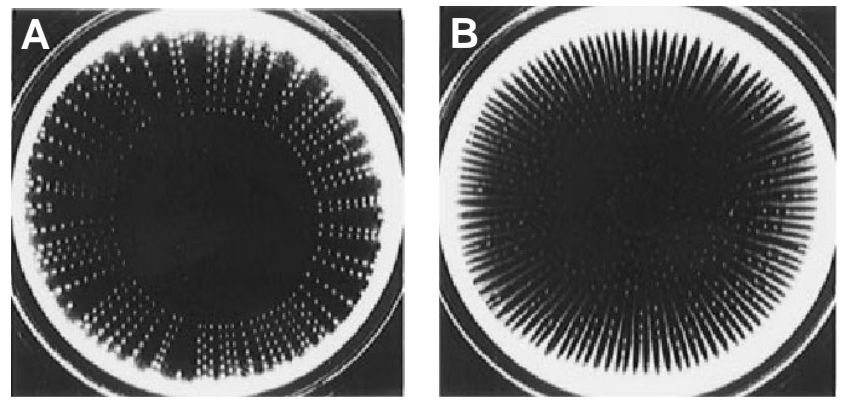

Fig. 1. Two examples of patterns of bacteria $E$. coliin experiments by Budrene and Berg (1991). Radial alignment of spots (A) and radially oriented stripes (B).

A number of mathematical models have been developed in order to explain the formation of ordered spatial patterns in colonies of motile bacteria. The model for pattern formation in bacterial colonies, suggested by Budriene et al. (1988), was based on the essential assumption of the delay in the cell response to external signals. This model was able to explain the formation of consequent concentric rings of high cell density in the course of bacterial colony growth, but failed to explain more complex patterns observed in experiments. The reason of this failure is quite clear from the present-day point of view: chemotactic response of bacteria to the gradients of attractants, produced by the cells themselves, was not taken into account, as its significance for pattern formation has been brought out clearly later (Budrene and Berg, 1991, 1995). The role of chemotaxis in pattern formation by motile bacteria was examined in a number of consequent models (Bruno, 1992; Woodward et al., 1995; BenJacob et al., 1995; Tsimring et al., 1995; Tyson, 1996; Brenner et al., 1998; Mittal et al., 2003). Woodward with colleagues (1995) suggested a model which successfully explained the patterns formed by motile $S$. typhimurium. However, the kinetics of formation and the very structures in this case are much different from those observed in E. colicolony, in particular, pattern formation is preceded by the uniform spread of bacteria all over the Petri dish, which noticeably simplifies the shape of the model.

In other models in order to reproduce numerically the observed patterns some rather artificial assumptions were made, such as existence of a second repellent field or the autocatalytic production of attractant triggered by waste (Ben-Jacob et al., 1995;

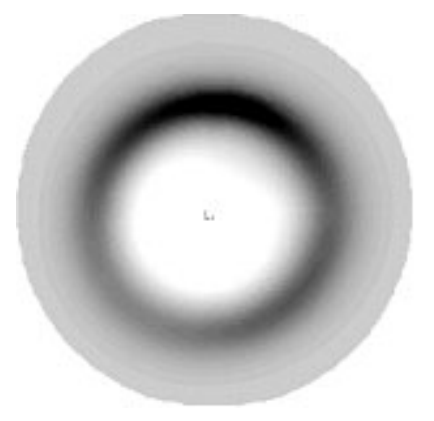

Fig. 2. The moving ring of vegetative cells. Black color corresponds to high cell density. Parameters used in simulations: $\alpha=16, \mu=2, \mathrm{~B}=8, \mathrm{~A}$ $=20, \mathrm{n} \lim =10, \mathrm{G}_{0}=0.65, \mathrm{D}_{n}=0.4, \mathrm{Ds}=\mathrm{D}_{c}=1, \gamma=0.05, \sigma=0.1$, $\mathrm{S}^{*}=0.1867, \mathrm{~S}=0.25, \mathrm{~N}=1.5$.
Tsimring et al., 1995) which were not supported by experimental data. Because all of these studies produced pictures that look qualitatively like the experiments, it is unclear which specific features are responsible for the pattern formation.

A qualitative step in the understanding the nature of complex patterns formed by motile $E$. colicells was made by Brenner et $a$. (1998), who systematically analyzed the mechanisms of bacterial interaction and outlined the basic principles of modeling.

In the present paper we will first discuss in more detail experimental data, then outline the basic principles of the model and formulate the corresponding equations. Finally we will present results of numeric simulations and compare them with experimental observations.

\section{Experimental background}

Under normal conditions, an E. colicell consists of an elongated body, to which several flagella are attached. Each flagellum is propelled by a rotary motor. There are two modes of operation of this motor, clockwise and counterclockwise. When the individual flagella rotate counterclockwise, they form a bundle and this bundle propels the bacterium forward; when the flagellum turns clockwise, the motions of the individual flagella are independent of each other, causing the cell to randomly change its orientation (Berg and Brown, 1972).

The environment must contain chemicals so that the bacterium can live and function normally. Bacteria require a carbon source, an energy source and inorganic salts. Typical assays for motility are engineered so that there are sufficient nutrients for normal survival; if, however, these nutrients are used up, there can be transitions in the internal state of the bacteria, affecting their motion. A common environmental response is chemotaxis (Pfeffer, 1884; Stock and Surette, 1996), in which cells move up an external chemical gradient. For $E$. coli, chemotaxis occurs by constant sampling of attractant as they move. Careful measurements demonstrate that the bacteria compute a weighted difference between the amount of attractant that binds to their receptors during the previous second of motion and the amount of attractant that has bound during the three preceding seconds (Segall et al., 1986). The weighting function used for this computation was directly measured in impulse response experiments on single bacteria. When the convolution of the weighting function with a stimulus is positive, the probability of tumbling decreases; this effectively increases the length of runs in directions of increasing attractant gradient.

The combined effect of the physiological and chemotactic responses of the bacteria motion results in nontrivial collective behaviors, which have been the focus of inquiry since Adler's introduction of assays in which E. colimove in migrating "bands". In Adler's experiments, the bands form when cells of $E$. coliare placed in an environment containing substances (oxygen, amino acids, etc.) that the bacteria both consume and respond to chemotactically. The consumption of the substance generates an attractant gradient, which provokes chemotaxis. The net response is a well-defined band of cells moving across a capillary tube or a Petri dish (Adler, 1966, 1969).

Budrene and Berg $(1991,1995)$ found conditions in which more complex patterns can form in an environment that is chemotactically inert. In contrast to Adler's experiments, the environ- 
A

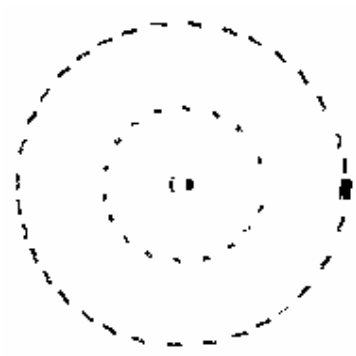

B

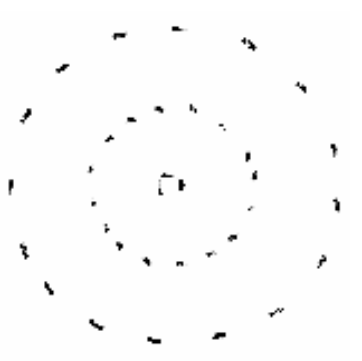

Fig. 3. Examples of patterns obtained numerically. (A) $A$ radial pattern. Parameters used in simulations: $\alpha=16, \mu=2, \mathrm{~B}=8, \mathrm{~A}=20, \mathrm{n} / \mathrm{lm}$ $=9, \mathrm{G}_{0}=0.65, \mathrm{D}_{n}=0.4, \mathrm{D} s=\mathrm{D}_{c}=1, \gamma=0.11, \sigma=0.1, \mathrm{~s}^{*}=0.225, \mathrm{~S}=0.8$, $\mathrm{N}=0.95$. (B) A hehagonal pattern. Parameters used are the same ones but $\mathrm{n} \lim =10, \mathrm{~s}^{*}=0.1867, \mathrm{~s}=0.35$.

mental conditions induce the bacteria to excrete an attractant (aspartate) toward which they undergo chemotaxis. The excretion of attractant means that there is effectively a long-range interaction among the bacteria. These conditions produce patterns that are dramatically different from Adler's initial experiments. Typical experiments are performed on agar plates, in which the agar concentration is low enough that the bacteria can move freely. The type of pattern depends strongly on the amount of a single carbon and energy source (succinate) that is uniformly distributed in the dish. Exposure to succinate is required for bacteria to perform intracellular reactions producing the attractant. At low succinate concentrations, the bacteria originate in the center of the Petri dish and form a swarm ring that propagates toward the boundary. At higher succinate concentrations the swarm ring destabilizes and produces a symmetrical array of dense compact structures called aggregates. When cells are grown in semi-solid agar they form symmetrical arrays of spots or stripes that arise sequentially. On the other hand, when cells are grown in a thin layer of liquid medium on a single carbon source, spots appear synchronously, more randomly arrayed. Microscopic examinations of the growing colonies revealed at least two morphologically distinct bacterial states: classic rod shaped motile cells (we will refer to them as vegetative bacteria) in the peripheral area and clusters of non-motile cells (referred to as anabiotic cells) in the central area of the colony.

\section{The model}

Now we will formulate a mathematical model based on the assumptions, which follow from the experimental data mentioned above. Our aim is to reveal the most essential properties of the bacterial colony which ensure the formation of patterns, observed by Budrene and Berg $(1991,1995)$, thus the model is to be as simple as possible.

The experiments described above suggest that the developing structure is formed by clusters of non-motile non-dividing cells. In other words, this structure is neither dynamic nor self-sustaining (in contrast to a dissipative structure). In all instances the pattern results from the propagation of a pulsatile wave of bacterial biomass growth. The passage of this wave leads to a non-uniform spatial distribution of cell density which is fixed due to the transition of vegetative bacteria to clusters of anabiotic bacteria. Thus the time-related constancy of the resulting spatial structure is due to the non-motility of clusters and cessation of division of the constituent bacteria. In fact the pattern is laid down on the moving front of the growing bacterial colony due to the instability of vegetative cell distribution. This instability is the result of the chemotactic response of bacteria to the attractant (aspartate), produced by the vegetative cells themselves. The rate of the attractant production depends on the amount of a single carbon and energy source (succinate), consumed by bacteria.

Thus the essential variables of the model are densities of vegetative $(n)$ and anabiotic ( $n \eta$ cells, attractant $(c)$ and nutrient $(s)$ concentrations. The corresponding model has the following form:

$$
\frac{\partial n}{\partial t}=\frac{G_{0} s}{s+\sigma} n-\gamma n \Theta(n) \Theta\left(n+n_{1}-n^{\lim }\right)-\alpha \nabla\left(\frac{n}{(c+\mu)^{2}} \nabla c\right)+D_{n} \nabla^{2} n,
$$

$$
\begin{aligned}
& \frac{\partial n_{1}}{\partial n}=\gamma n \Theta(n) \Theta\left(n+n_{1}-n^{\lim }\right), \\
& \frac{\partial c}{\partial t}=A(s) n-B c+D_{c} \nabla^{2} c \\
& \frac{\partial s}{\partial t}=-\frac{G_{0} s}{s+\sigma} n+D s \nabla^{2} s, \\
& A(s)= \begin{cases}A_{0} s, & s \geq s^{*} \\
A_{0} s^{*}, & s<s^{*}\end{cases}
\end{aligned}
$$

In Eq. (1) the term $G_{0} s n /(s+\sigma)$, corresponding to cell division, takes into account two experimental facts: the slowing down of the growth rate for low nutrient concentrations and its finite quantity for high concentrations. The term $\gamma n \mathrm{Q}(n) \mathrm{Q}\left(n+n_{1}-n^{\mathrm{lim}}\right)$, entering Eqs. (1) and (2), describes the transition of vegetative cells into anabiotic form due to the increase of local cell density. The transition starts when the total cell density reaches the value $\mathrm{nim}$. In the equations it is ensured by the corresponding Heaviside function $\mathrm{Q}\left(n+n_{1}-n^{\lim }\right)$.

Two other terms in Eq. (1) describe cell motility, both random, with the motility coefficient $D$ nand chemotactic. Let's consider the chemotactic term $-\alpha \nabla\left(n /(c+\mu)^{2} \star \nabla c\right)$ in more detail. As is known from experiments, the chemotactic cell response depends on the

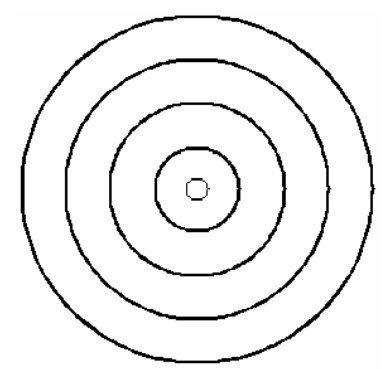

Fig. 4. Concentric stationary rings formed by aggregated cells. Parameters used in simulations: $\alpha=16, \mu=2, \mathrm{~B}=8, \mathrm{~A}=20, \mathrm{n} \lim =9, \mathrm{G}_{0}$ $=0.65, \mathrm{D}_{n}=0.4, \mathrm{D} s=\mathrm{D}_{c}=1, \gamma=3, \sigma=0.1, \mathrm{~s}^{*}=0.18, \mathrm{~S}=1, \mathrm{~N}=0.95$. 


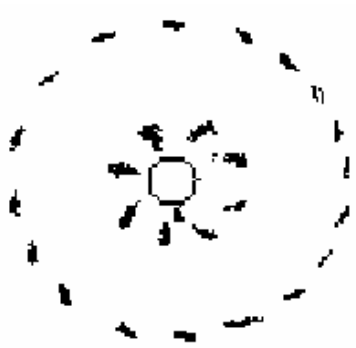

Fig. 5. Elongated cell aggregates (stripes). Parameters used in simulations: $\alpha=16, \mu=2, \mathrm{~B}=8, \mathrm{~A}=20, \mathrm{n} / \mathrm{im}=15, \mathrm{G}_{0}=0.8, \mathrm{D}_{n}=0.4, \mathrm{D} s=$ $\mathrm{D}_{c}=1, \gamma=3, \sigma=0.1, \mathrm{~S}^{*}=0.36, \mathrm{~S}=0.5, \mathrm{~N}=1.15$.

attractant receptor occupation (Brown and Berg, 1974). Here $\mu$ is the dissociation constant of the receptor. For low attractant concentration $(c<<\mu)$ chemotactic velocity is proportional to the attractant concentration gradient $\nabla c$ with the proportionality coefficient $\alpha$. With the increase of the attractant concentration the cellular sensitivity to the attractant gradient significantly decreases.

Equation (3) describes attractant production, decay and diffusion. The essential assumption is that its production by vegetative cells depends on the nutrient concentration (Brenner etal., 1998). Below a certain basic nutrient level $s^{*}$, its rate is constant, while above this level it grows with the nutrient concentration.

The last equation describes evolution of the nutrient distribution depending on its consumption by bacteria and on diffusion.

\section{Results}

The model, formulated above, was treated both analytically and numerically. In the linear analysis, given in the Appendix, the stability of the expanding ring on the edge of the growing bacterial colony was examined. As a result the conditions for parameters were obtained, which correspond to the patterns observed in experiments. These values of parameters were used in calculations.

Numerical simulations of Eqs. (1)-(4) were performed in the polar reference frame using the method of splitting related to physical processes in the domain $0<R R, 0<\varphi<2 \pi$, where $\mathrm{R}$ is the radius of the Petri dish. For the initial conditions the following values were taken: $n=N$ in $0<r<R_{0}, 0<\varphi<2 \pi$ and $s=S, c=$
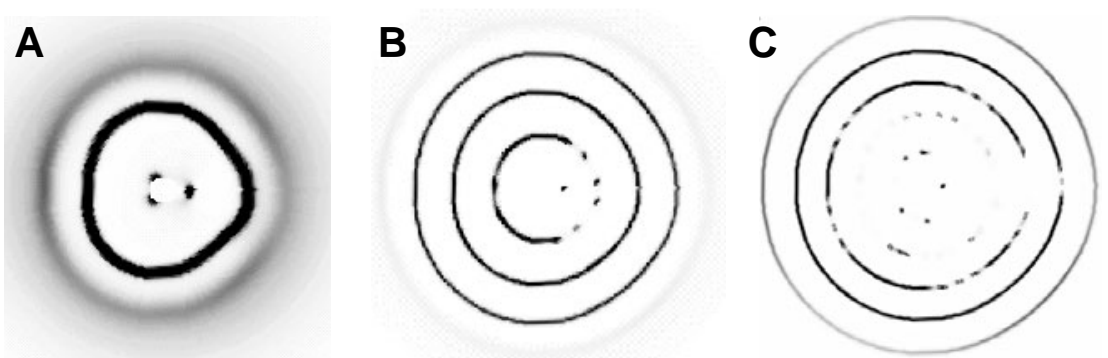

Fig. 6. The dynamics of pattern formation (see details in the text). Parameters used in simulations: $\alpha=16, \mu=2, \mathrm{~B}=8, \mathrm{~A}=20, \mathrm{n} \lim =15, \mathrm{G}_{0}=0.8, \mathrm{D}_{n}=0.4, \mathrm{D} s=\mathrm{D}_{c}=1, \gamma=$ $3, \sigma=0.1, \mathrm{~s}^{*}=0.36, \mathrm{~S}=0.5, \mathrm{~N}=1.15$. $n 1=0$ in $0<r<R, 0<\varphi<2 \pi$, where $R_{0}$ is the radius of inoculation. In the area of inoculation the initial level of bacterial density $N$ was disturbed by a random noise. For the boundary conditions zero fluxes at $r=0, r=R$ were taken.

Numerical experiments have shown that the main parameters, influencing the type of the emerging pattern are the initial cell density $N$ and nutrient concentration $\mathcal{S}$, chemotaxis strength $\alpha$, the threshold for attractant production $s^{\star}$ and the rate of transition of vegetative cells into anabiotic form $\gamma$. By variation of these parameters it appeared possible to obtain numerically patterns observed in experiments and to investigate the dynamics of their formation.

For low nutrient concentrations the cell density in the moving ring does not reach the threshold value for the transition into the anabiotic state. The attractant concentration is not sufficient for the break of stability of the uniform cell distribution along the ring. Thus, the latter moves in an autowave fashion (Fig. 2).

For higher initial nutrient concentration cell density in the expanding ring becomes sufficient both for the break of stability of the uniform cell distribution and for their aggregation. In particular, if after the formation of the successive set of aggregates the expanding ring had time to grasp a certain part of the cells, participating in aggregation, then a radial pattern is formed (Fig. $3 A)$. Aggregates of the next set appear both opposite the previous ones and between them. On the other hand, if the expanding ring had no time to grasp cells from the previous set of aggregates, the hexagonal pattern is formed (Fig. 3B). In this case aggregates of the net set appear only between the previous ones.

If the rate of cell transition into anabiotic state is increased, the attractant production is not sufficient for the ring segmentation. Thus the emerging pattern has the shape of stationary concentric cell rings (Fig. 4).

In the case when cells have not completely consumed nutrients behind the moving edge of the bacterial colony, after aggregation they start to move in the reverse direction. Simultaneously some of them transform into the anabiotic state and thus the stripes are formed (Fig. 5).

In Fig. 6 the dynamics of aggregate formation is observed. A part of cells from the expanding ring stops, while the rest ones continue to move forward (Fig. 6A). In a certain moment, when the attractant concentration reaches the critical value, the stationary ring breaks into separate aggregates (Fig. 6B). This process repeatedly continues till the edge of the Petri dish is reached (Fig. $6 C)$.

Thus the results of numerical experiments demonstrate that the model, suggested in the present paper, quite adequately reflects the mechanism of intercellular regulation in the bacterial colony, causing the formation of complex spatial patterns. By variation of the model parameters it appeared possible both to reproduce numerically the patterns, observed in experiments and to examine the dynamics of their formation.

\section{Acknowledgments}

This work was supported by grants from the Russian Foundation of Basic Research and by the grant of support for leading scientific schools of Russia. 


\section{References}

ADLER, J. (1966). Chemotaxis in bacteria. Science. 153:708-716.

ADLER, J. (1969). Chemoreceptors in bacteria. Science. 166:1588-1597.

AGLADZE, K., BUDRIENE, E., IVANITSKY, G., KRINSKY, V., SHAKHBAZYAN, V. and TSYGANOV, M. (1993). Wave mechanisms of pattern formation in microbial populations. Proc. R. Soc. Lond. B. Biol. Sci. 253: 131-135.

ALT, W. and LAUFFENBURGER, D.A. (1987). Transient behavior of a chemotaxis system modeling certain types of tissue inflammation. J. Math. Biol. 16:141-163.

BEN-JACOB, E., COHEN, I., SHOCHET, O., ARANSON, I., LEVINE, H. and TSIMRING. L. (1995). Complex bacterial patterns. Nature. 373:566-567.

BERG, H. C. and BROWN, D. A. (1972). Chemotaxis in Escherichia colianalysed by three dimensional tracking. Nature. 239:500-504.

BONNER, J. (1967). The Cellular Slime Molds. Princeton University Press, Princeton, $\mathrm{NJ}, 205 \mathrm{pp}$.

BRENNER, M. P., LEVITOV, L. S. and BUDRENE, E. O. (1998). Physical mechanisms for chemotactic pattern formation by bacteria. Biophys. J. 74:1677-1693.

BROWN, D.A. and BERG, H.C. (1974). Temporal stimulation of chemotaxis in Escherichia coli. Proc. R. Soc. Lond. B. Biol. Sci. 71: 1388-1392.

BRUNO, W. (1992). CNLS Newsletter. 82:1-10.

BUDRENE, E. and BERG, H. (1991). Complex patterns formed by motile cells of Escherichia coli. Nature. 349:630-633.

BUDRENE, E. and BERG, H. (1995). Dynamics of formation of symmetrical patterns by chemotactic bacteria. Nature. 376:49-53.

BUDRIENE, E.O., POLEZHAEV, A.A. and PTITSYN, M.O. (1988). Mathematical modelling of intercellular regulation causing the formation of spatial structures in bacterial colonies. J. theor. Biol. 135:323-341.

HOUSER, G. (1885). Uber Fäulmisslakterien und deren Beriehungen zur Septicämie. Leipzig, F.G.V. Vogel.

KELLER, E.F. and SEGEL, L.A. (1970). Initiation of slime mold aggregation viewed as an instability. J. Theor. Biol. 26:399-415.

KELLER, E.F. and SEGEL, L.A. (1971). Travelling bands of chemotaxis system modeling certain types of tissue inflammation. J. Math. Biol. 16: 141-163.

LAUFFENBURGER, D.A. and KENNEDY, C.R. (1983). Localized bacterial infec- tion a distributed model for tissue inflammation. J. Math. Biol. 24: 691-722.

MITTAL, N., BUDRENE, E.O., BRENNER, M. P. and VAN OUDENAARDEN, A. (2003). Motility of Escherichia coli cells in clusters formed by chemotactic aggregation. Proc. Natl. Acad. Sci. USA. 100: 13259 - 13263.

MURRAY, J.D. (1989). Mathematical Biology. Berlin, Springer-Verlag. [2nd corrected edition 1993]. $760 \mathrm{pp}$.

MURRAY, J.D., DEEMING, D.C. and FERGUSON, M.W.J. (1990). Size-dependent pigmentation-pattern formation in embryos of Alligator mississipiensis: time of initiation of pattern generation mechanisms. Proc. R. Soc. Lond. B. Biol. Sci. 239: 279-293

MURRAY, J.D. and MYERSCOUGH, M.R. (1991). Pigmentation pattern formation on snakes. J. Theor. Biol. 149: 339-360.

NANJUNDIAH, V. (1973). Chemotaxis, signal relaying and aggregation morphology. J. Theor. Biol. 30: 63-105.

NOSSAL, R. (1972). Growth and movement of rings of chemotactic bacteria. Exp. Cell. Res. 75: 138-142.

OSTER, G.F. and MURRAY, J.D. (1989). Patterns formation models and developmental constrains. J. Exp. Zool. 251:186-202.

PFEFFER, W. (1884). Untersuch. Bot. Inst. Tubingen. 1:363.

SEGALL, J. E., BLOCK, S. and BERG, H. C. (1986). Temporal comparisons in bacterial chemotaxis. Proc. Natl. Acad. Sci. USA. 83:8987-8991.

STOCK, J. B. and SURETTE, M. G. (1996). Chemotaxis. In Escherichia coli and Salmonel/a. F. C. Neidardt, editor. ASM Press, Washington, DC. 1103-1129.

TSIMRING, L., LEVINE, H., ARANSON, I., JACOB, E.B., COHEN, I., SHOCHET, O. and REYNOLDS, W. (1995). Aggregation patterns in stressed bacteria. Phys. Rev. Lett. 75:1859-1862.

TYSON, R. (1996). Pattern formation by E. coli mathematical and numerical investigation of a biological phenomenon. Ph.D. thesis. University of Washington, Seattle.

WOLFE, A. J. and BERG, H. (1989). Migration of bacteria in semi-solid agar. Proc. Natl. Acad. Sci. USA. 86:6973-6977.

WOODWARD, D. E., TYSON, R., MYERSCOUGH, M., MURRAY, J. D., BUDRENE, E. and BERG, H. (1995). Spatio-temporal patterns generated by Salmonella typhimurium. Biophys. J. 68:2181-2189.

\section{Mathematical Appendix}

Here we obtain the conditions for instability of the expanding ring on the front of the growing colony. As the total cell density is small there we disregard the transition of vegetative cells into anabiotic form. We also neglect the first term in Eq. (1) assuming that the characteristic tine of cell division is large compared with that of the cell motility. Then Eqs. (1), (3) take the following form:

$$
\begin{aligned}
& \frac{\partial n}{\partial t}=-\alpha \nabla\left(\frac{n}{(c+\mu)^{2}} \nabla n\right)+D_{n} \nabla^{2} n, \\
& \frac{\partial c}{\partial t}=A(s) n-B c+D_{c} \nabla^{2} c .
\end{aligned}
$$

Linearizing Eqs. (A1), (A2) near the uniform steady state $\left(n_{0}, c_{0}\right)$, where $A(s) n_{0}=B c_{0}$, we obtain

$$
\frac{\partial n}{\partial t}=-\left(\frac{\alpha n_{0}}{\left(c_{0}+\mu\right)^{2}}\right) \nabla^{2} n+D_{n} \nabla^{2} n
$$

$$
\frac{\partial c}{\partial t}=A(s) n-B c+D_{c} \nabla^{2} c
$$

Here $n$ and $c$ are deviations from the steady state $n_{0}$ and $c_{0}$ respectively. Taking them in the form $\left(\begin{array}{l}\delta n \\ \delta c\end{array}\right) e^{i \bar{k} \bar{x}+\lambda t}$, we arrive at the following dispersion equation:

$$
\lambda^{2}+\left[k^{2}\left(D_{n}+1\right)+B\right] \lambda+k^{2} D_{n}\left(k^{2}+B\right)-\frac{A(s) \alpha n_{0} k^{2}}{\left(\mu+c_{0}\right)^{2}}=0 .
$$

The uniform state is unstable due to the bacterial motility, if there exists an interval of wave numbers $k^{2}$ for which one of the eigenvalues of Eq. (A5) is positive. It can be so if the free term of Eq. (A5) is negative: 


$$
D_{n}\left(k^{2}+B\right)-\frac{A(s) \alpha n_{0}}{\left(\mu+c_{0}\right)^{2}}<0,
$$

or, using the relation, $A(s) n_{0}=B c_{0}$,

$$
D_{n}\left(k^{2}+B\right)-\frac{B \alpha c_{0}}{\left(\mu+c_{0}\right)^{2}}<0 .
$$

It is possible if

$$
\frac{c_{0}}{\left(c_{0}+\mu\right)^{2}}>\frac{D_{n}}{\alpha} \text {. }
$$

This inequality can be presented in the form

$$
f(c)=c_{0}^{2}+\left(2 \mu-\frac{\alpha}{D_{n}}\right) c_{0}+\mu^{2}<0
$$

Minimum of the function $l(c)$ is negative if

$$
-\frac{1}{4}\left(2 \mu-\frac{\alpha}{D_{n}}\right)^{2}+\mu^{2}<0,
$$

which gives the condition

$$
\alpha>4 \mu D_{n} \text {. }
$$

Now let's estimate the value of $s^{*}$. From Eqs. (1) and (4) it follows that for the uniform distribution $n_{0}=S-s_{0}$, where $\left(n_{0}, s_{0}\right)$ is the quasi-stationary state and $S$ is the initial nutrient concentration. From the condition $A(s) n_{0}=B c_{0}$ it follows, that

$c_{0}=\frac{A(s)}{B} n_{0}=\frac{A(s)}{B}\left(S-s_{0}\right) \rightarrow \frac{A_{0} s^{*} S}{B}$, for $s_{0} \rightarrow 0$.

Substituting (A9) into (A6) we obtain

$\frac{A_{0} s^{*} S B^{2}}{B\left(A_{0} s^{*} S+B \mu\right)^{2}}>\frac{D_{n}}{\alpha}$,

or

$$
s^{* 2}+2 s^{*} \frac{B}{A_{0} S}\left(2 \mu-\frac{\alpha}{D_{n}}\right)+\left(\frac{\mu B}{A_{0} S}\right)^{2}<0 .
$$

The roots of this square equation are

$$
s_{1,2}^{*}=\frac{1}{2} \frac{B}{A_{0} S}\left(\frac{\alpha}{D_{n}}-2 \mu \pm \sqrt{\frac{\alpha^{2}}{D_{n}^{2}}-\frac{4 \mu \alpha}{D_{n}}}\right) .
$$

Thus the required value of $s^{*}$ lies in the interval between these roots. 